\title{
LA MESURE DE L'EAU TOTALE DE L'ORGANISME CHEZ LES PORCINS PAR LA MÉTHODE A L'ANTIPYRINE
}

PAR

\section{B.-L. DUMONT ${ }^{(1)}$}

Station de Recherches sur 1'Élevage, C.N.R.Z., Jouy-en-Josas.

La mesure de l'eau totale de l'organisme, par la méthode des espaces de diffusion, a donné lieu, ces dernières années, à de nombreuses études.

Trois indicateurs principaux ont retenu l'attention des chercheurs :

l'eau lourde $\left(\mathrm{D}_{2} \mathrm{O}\right)$.

l'eau tritiée ('THO).

l'antipyrine (I-phényl-2,3 diméthylpyrazolone 5 one).

I,es deux premières substances se comportent, dans l'organisme, sensiblement comme de l'eau ordinaire et présentent, de ce fait, un avantage certain sur l'antipyrine.

Mais leur coût et les difficultés que présente leur dosage limitent pour l'instant leur usage systématique dans de nombreux travaux de recherches.

L'utilisation de l'antipyrine, introduite par SoBERMAN et coll. (I), qui en ont étudié la diffusion dans les différents tissus de l'homme, permet de pallier ces deux difficultés. L'antipyrine répond assez bien, dans l'espèce humaine tout au moins, aux conditions imposées à un indicateur d'espace de diffusion :

distribution rapide et identique dans tout le corps,

non-toxicité,

élimination lente (transformation lente dans l'organisme, excrétion lente hors de l'organisme),

dosage facile et simple.

I a méthode à l'antipyrine a été appliquée, depuis, à la mesure de la masse hydrique de nombreuses espèces.

Elle a été utilisée notamment par KRAYBiLL et coll. (2), dans leurs travaux sur la composition corporelle des porcs. Ces auteurs concluent

(I) Ce travail a été réalisé avec l'assistance de II. Erwin Kvoertzer et la collaboration technique de Mlle Éléonora Hunzik, MM. Jean RetTagliati et Jean Weber. 
que "les mesures de l'eau corporelle réalisées " in vivo " grâce à la méthode à l'antipyrine, fournissent l'une des techniques les plus récențes et les plus utiles pour déterminer la composition corporelle ", bien que 1'antipyrine présentât, chez le porc, une élimination assez rapide ( 36 p. IoJ par heure).

Depuis ce travail, cette technique n'a pas été appliquée, en dépit de l'intérêt que lui prêtent ses auteurs. Aussi bien, avant d'utiliser cette méthode d'une façon systématique, avons-nous voulu en étudier le détail et préciser les conditions de sa réalisation. 'Tel est l'objet du travail relaté ici.

\section{Technique expérimentale}

L'étude a porté sur 73 porcs, principalement de race Large White, de 80 à Ioo kg de poids vif. Treate de ces animaux furent utilisés uniquement pour suivre la diffusion de la substance dans différents tissus.

Tous les animaux étaient à jeun de I2 heures au moment de l'injection.

Les injections d'antipyrine furent faites dans l'une des veines de l'oreille, au moyen d'une seringue calibrée. La dose, variable selon les cas, était diluée dans du sérum physiologique, sous $30 \mathrm{~cm}^{3}$. de volume.

Les prises de sang étaient effectuées à la veine cave antérieure, selon une méthode précédemment décrite (3).

Le sang était recueilli sur héparine et centrifugé ; le plasma déféqué selon la méthode de BRODIE (4), par déprotéinisation zincique. Le dosage de l'antipyrine fut pratique selon la méthode de BRoDre, modifiéa par nos soins (5). La technique d'extraction de l'antipyrine tissulaire utilisée fut celle proposée par Brodie (4).

\section{Résultats}

\section{I. - Caractères de la courbe d'élimination}

Après son introduction dans l'organisme, l'antipyrine disparaît rapidement du sang. I,es graphiques (fig. I-2) illustrent ce phénomène. Ils indiquent les variations en fonction du temps après injection :

de la densité optique totale du sang/nitrite (fig. I),

de la concentration du plasma en antipyrine (fig. 2):

Les variations observées dépendent:

des différences dans les doses injectées (fig. 3),

des différences entre porcs, toutes conditions étant égales par ailleurs (même poids, même dose) (fig. 4). La variation individuelle masque en partie l'influence de la dose sur la courbe d'élimination. 
Le pourcentage de disparition horaire de 1'antipyrine, obtenu par le rapport:

Concentration plasmatique en antipyrine à tr - Concent. plasm. en antipyrine à tz concentration plasmatique en antipyrine à $\mathrm{t} i$

(tI, t2, t $n$ étant la première, la deuxième, la $n^{\mathbf{e}}$ heure après injection)

peut caractériser la vitesse de disparition plasmatique de la substance. Il est en moyenne de 6I,35 p. Ioo entre la $\mathrm{I}^{\text {ere }}$ et la $2^{\mathrm{e}}$ heure après injection (valeurs extrêmes: $5^{0}-85$ p. Ioo) et de $6 \mathrm{I}, 75 \mathrm{p}$. Ioo entre la $2^{\mathrm{e}}$ et $1 \mathrm{a}$ $3^{\text {e }}$ heure.

Cette valeur est la plus élevée qui ait été obtenue pour la vitesse d'élimination de l'antipyrine chez les différentes espèces étudiées jusqu'ici. Elle diffère nettement de celle avancée par KRAYBIL. ( 36 p. Ioo).

Cette élimination rapide altère fortement l'interprétation que l'on peut faire de la courbe de disparition plasmatique de l'antipyrine. La limite entre la période de diffusion et la période d'élimination, qui d'ordinaire se caractérisent par des pentes différentes sur la courbe, est difficile à préciser.

En coordonnées semilogarithmiques, la courbe d'élimination n'est assimilable à une droite que pendant un temps relativement court, et dans ce cas, la méthode d'extrapolation proposée par CACHERA et BARBIER (6) ne s'applique, d'après les résultats obtenus chez le porc, que lorsque les concentrations plasmatiques restent suffisantes. La plupart du temps, 2 heures $\mathrm{r} / 2$ après injection, les points obtenus ne sont pas alignables sur la droite (fig. 5).

On comprend, en raison du choix de l'échelle logarithmique pour les ordonnées, que l'ajustement à la droite d'élimination ne soit plus possible après un certain temps, lorsque les concentrations observées deviennent faibles.

En outre, les points obtenus en fin d'élimination correspondent à de faibles quantités d'antipyrine, sur la détermination desquelles les erreurs relatives peuvent être fortes. Là encore, le choix de l'échelle logarithmique peut expliquer en partie les écarts linéaires importants enregistrés entre les valeurs observées en fin d'élimination et celles correspondant à la droite théorique d'élimination.

Quatre points sont nécessaires pour établir la courbe et par suite, les 4 prélèvements correspondants sont à effectuer pendant l'intervalle de temps compris entre 30 minutes et 2 heures après injection (et non à $2 \mathrm{~h}$. 30 et $3 \mathrm{~h}$. après injection, comme le proposent KRAYBILL et coll. (2) ( $\left.{ }^{1}\right)$. L'étude de ces droites indique, d'autre part, qu'après 30 minutes

(1) Il est indispensable, en outre, d'opérer sur des animaux à jeun depuis $\mathbf{2} 2$ heures au moins. D'une part, pour obtenir le poids réel de l'animal avec précision, et d'autre part, pour supprimer l'interférence des produits de la digestion avec l'antipyrine plasmatique (fig. 6). L'absorption propre des sangs témoins prélevés sur des animaux à jeun depuis i 2 heures est pratiquement constante (fig. 7).

Annales de Zootechnie. - 1955 . 
et pour une forte dose injectée, les points sont alignés sur la droite d'élimination, ce qui indiquerait par suite une diffusion très rapide de l'antipyrine dans les différents tissus.

\section{2. - Quantité d'eau calculée}

I,es différents résultats obtenus figurent dans le graphique (fig. 8). Ils sont voisins de ceux de KraybiLi, (2).

\section{3. - Discussion. Précision de la méthode}

\section{a) Variabilité des résultats}

Les points expérimentaux obtenus ne sont jamais parfaitement alignables sur la droite d'élimination, en raison des erreurs diverses de manipulation. L'on est amené, le plus souvent, à aligner les points sur une droite d'ajustement théorique. L'erreur commise dans la détermination de la valeur de l'ordonnée au temps o (qui fournit la concentration initiale du plasma en antipyrine, en supposant que la diffusion est instantanée) varie de la même façon que la pente de la droite, c'està-dire comme une tangente. Plus la pente est forte, plus l'erreur est grande. Elle est d'autant plus importante qu'on a à considérer, en coordonnées logarithmiques, des valeurs élevées de concentration au temps o. C'est ce qui se passe dans le cas du porc, où les concentrations initiales dépassent roo $\gamma$ d'antipyrine par gramme d'eau plasmatique.

La grande vitesse d'élimination oblige, en effet, à injecter une dose importante d'antipyrine $(6 \mathrm{~g} / \mathrm{roo} \mathrm{kg}$ de poids vif) pour obtenir, 2 heures après injection, des quantités d'antipyrine plasmatique suffisamment fortes pour se prêter à un dosage précis.

Des écarts importants dans le pourcentage peuvent être obtenus en alignant les points de différentes façons (tableau I).

\section{TABLEAU I}

Ecart maximum entre les valeurs extrêmes obtenues par 2 ajustements et la valeur moyenne, en $\%$ de cette valeur moyenne.

\begin{tabular}{|c|c|c|c|c|c|c|c|c|c|c|}
\hline & $\circ$ & $1-2$ & $2-3$ & $3-4$ & +-5 & $5-6$ & $6-7$ & $7-8$ & 9 - I0 & $>10$ \\
\hline $\begin{array}{c}\text { Fréquence des } \\
\text { observations } .\end{array}$ & 2 & $t$ & 6 & 3 & 3 & & & 2 & I & 14 \\
\hline
\end{tabular}

Le tableau I indique la précision obtenue au cours des différents essais de cette étude. Les résultats consignés dans ce tableau comprennent : 
I $^{0}$ ceux qui ont été obtenus en appliquant la méthode originale de KRAYBILI, quant aux doses et aux heures de prélèvement. Ils portent sur I7 porcs, dont aucun ne donne une précision suffisante (tous ces résultats donnent un écart supérieur à 5 p. IOO);

$2^{0}$ ceux obtenus en pratiquant les prélèvements à des temps compris entre $I / 2$ heure et 2 heures après l'injection. Pour ceux-ci, au nombre de I 8 , la précision est meilleure (écart inférieur à 5 p. IOO).

\section{b) Signification des résultats - Variation dans la diffusion.}

En outre, le dépouillement des analyses des différents tissus semble indiquer que la répartition de l'antipyrine ne s'y fait pas d'une façon homogène.

La distribution de l'antipyrine fut étudiée sur 30 porcs de too $\mathrm{kg}$, ayant reçu la même dose $(6 \mathrm{~g})$ et abattus à des temps variables après injection et couvrant à la fois la période de diffusion et la période d'élimination.

Les tissus analysés furent le foie, le poumon, le cour, le diaphragme, le lard dorsal et le gras périrénal.

Le rapport :

$$
\frac{\text { antipyrine rapportée à l'eau tissulaire }}{\text { antipyrine rapportée à l'eau plasmatique }}
$$

fut retenu pour apprécier la diffusion tissulaire de la substance. Si la diffusion de l'antipyrine était homogène, ce rapport de diffusion serait de I.

En fait, la diffusion de l'antipyrine paraît hétérogène. Le rapport de diffusion est inférieur à I pour les différents tissus étudiés et s'en écarte même de façon sensible. Les moyennes obtenues dans ces tissus vont de 0,6 à 0,8 . Le cour et le poumon sont les tissus où la diffusion de la substance serait la moins importante, fait déjà signalé chez 1'hom me par Soberman et coll. (I).

En fin de la période d'élimination, la disparition de l'antipyrine tissulaire n'affecte pas de la même façon les divers tissus. Les tissus gras, lard et panne, conservent leur antipyrine plus longtemps et en plus forte proportion que les tissus viscéraux ou le muscle. Des rapports de diffusion supérieurs à I furent observés pour les tissus gras.

Les dosages effectués pendant la période de diffusion qui suit l'injection, confirment la diffusion très rapide de 1'antipyrine dans les tissus. En moins de 30 minutes pour tous les animaux étudiés, le rapport de diffusion atteignit sa valeur la plus élevée, indiquant par là la limite de la possibilité de diffusion de la substance. 

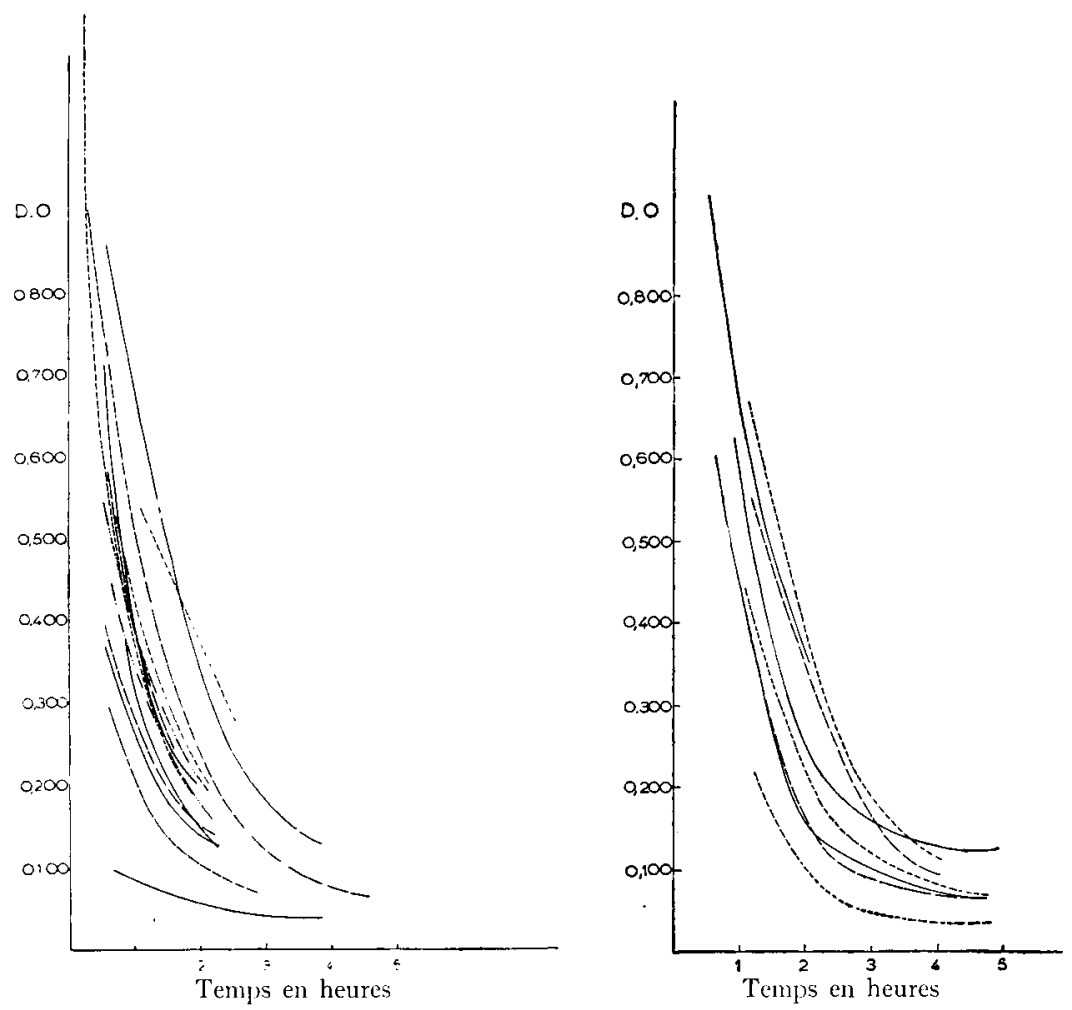

Fig. I. - Variation de la densité optique du sang par rapport au nitrite, en fonction du temps après injection.

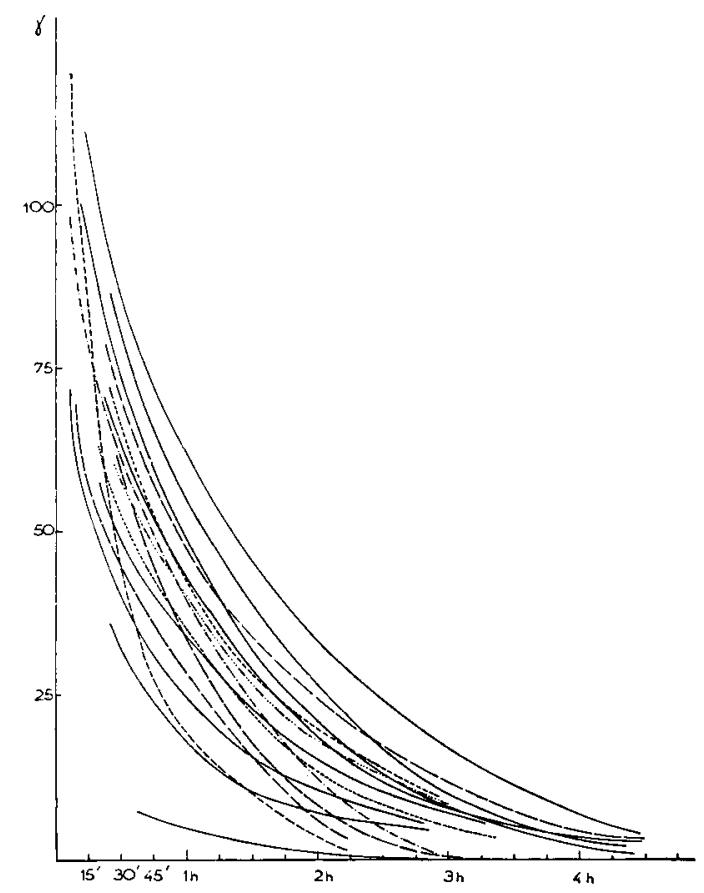

Fig. 2. - Variation de la concentration du plasma en antipyrine en fonction du temps après injection. 


\section{Conclusion}

Les deux caractéristiques présentées par la diffusion et l'élimination de cette substance chez le porc:

élimination très rapide,

diffusion inégale,

font que les mesures réalisées par l'emploi de cet indicateur reflètent mal l'importance réelle de la masse hydrique du porc. Pour des déterminations précises, l'emploi de l'antipyrine est à abandonner au profit d'indicateurs plus sûrs, l'oxyde de tritium par exemple, dont il est possible maintenant de faire un micro-dosage précis (7), mis au point par FALLOT, à l'époque où a été réalisée cette étude.

\section{RÉFÉRENCES BIBLIOGRAPHIQUES}

(I) Soberman (R.), Brodie (R. B.), Lévy (B. B.), Axelrod (J.), HolitanDER (V.), STEELE (J. M.). - J. Biol. Chem., 179, 3I, I949.

(2) Kraybili (H. F.), Goode (E. R.), Robertson (R. S. B.), Sloane (H. S.). - J. App. Phys., 6, 27-32, I953.

(3) Dumont (B.). - Ann. Zootechnie, IV, 297-303, I955.

(4) Brodie (B. B.), Axeirod (J.), Soberman (R. J.), Lévy. - J. Biol. Chem., 179, 25-30, I949.

(5) Dumont (B.). - Ann. Zootechnie, IV, 285-296, 1955.

(6) Cachera et Barbier. - In " Métabolisme de l'eau " de Hamburger et Mathe, Paris, I952.

(7) Faliót et Aeberhardt (A.). - Méthode de dosage du tritium (sous presse).

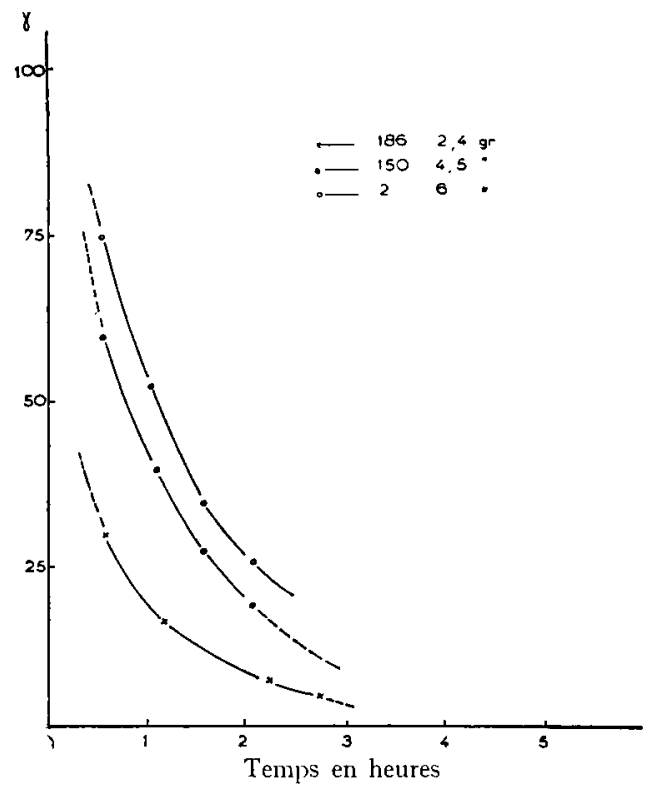

Fig. 3. - Influence de la dose injectée (Porc $n^{\circ}$ г86, 2,4 g; Porc $n^{0}$ I 50, 4,5 g; Porc $n^{\circ}$ 2,6g) sur la forme de la courbe d'élimination de l'antipyrine. 


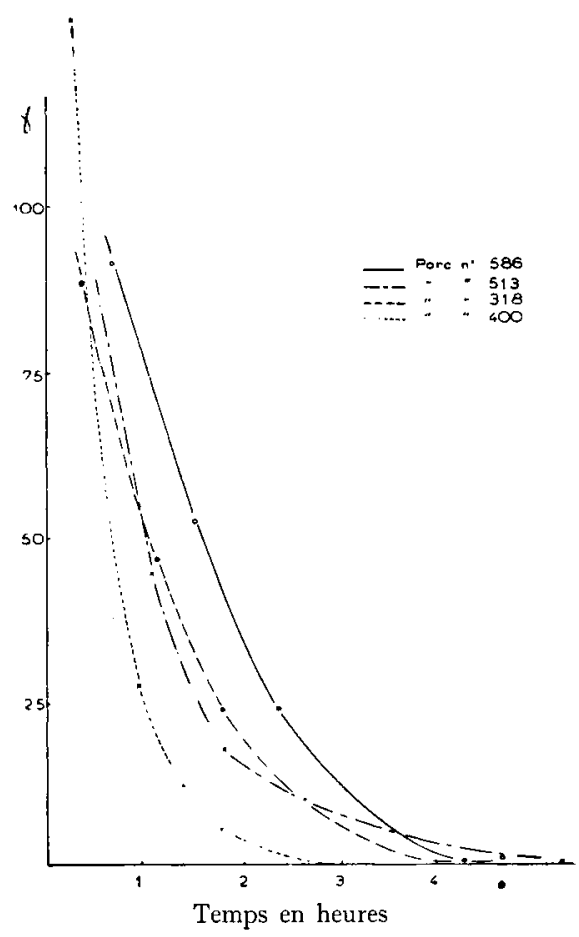

Fig. 4. - Variations individuelles dans la courbe d'élimination de l'antipyrine. (Les porcs, pesant chacun $89 \mathrm{~kg}$, ont tous reçu $6 \mathrm{~g}$ d'antipyrine).

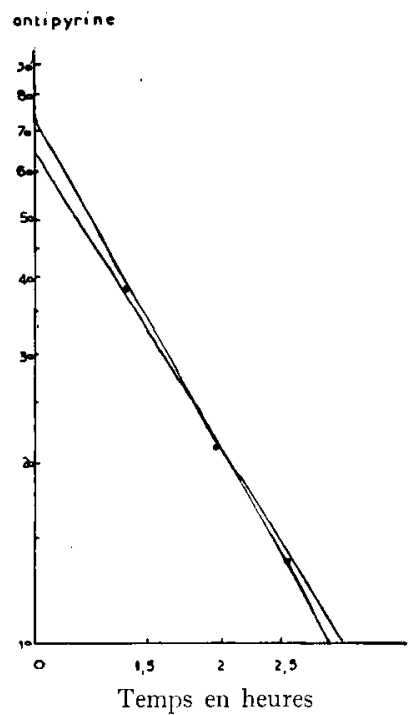

Fig. 5. - Courbe d'élimination de l'antipyrine en coordonnées semi-logarithmiques. 


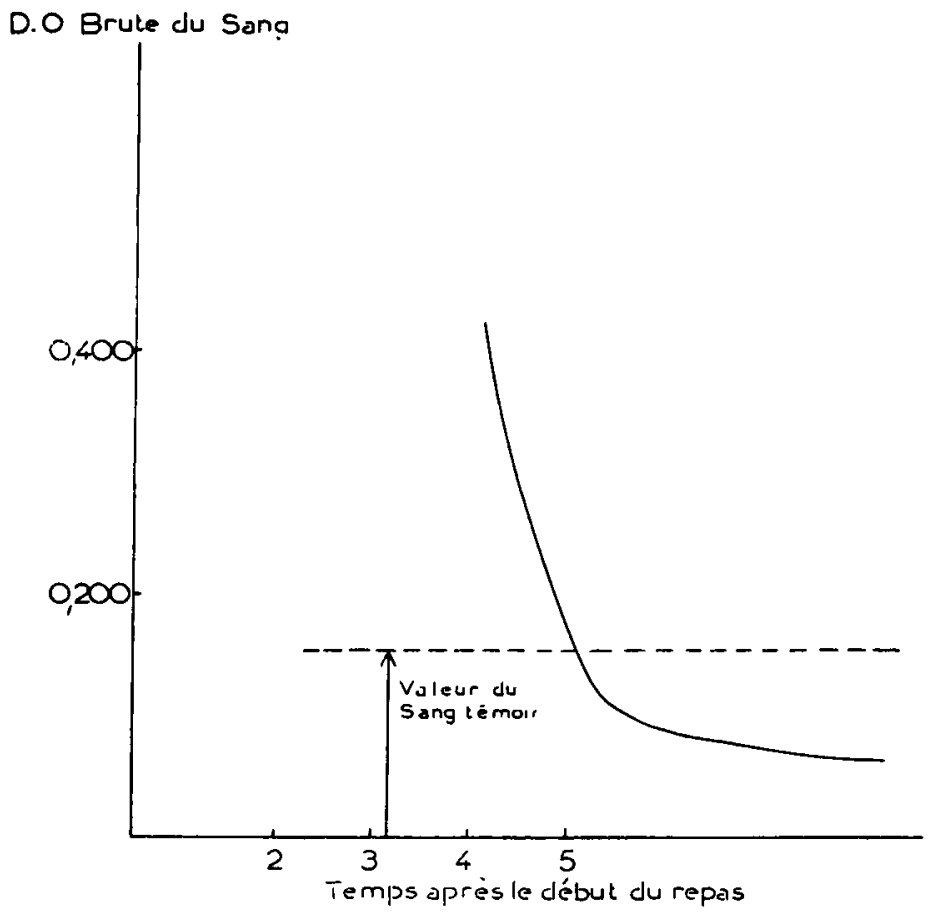

Fig. 6. - Variation de la densité optique du sang d'un porc ayant reçu une dose d'antipyrine $3 \mathrm{~h}$ ro après le début du repas. Les densités optiques mesurées 2 heures après l'injection sont nettement inférieures à la densité optique du sang témoin, prélevé avant injection. En ordonnées la densité optique du sang par rapport au nitrite.

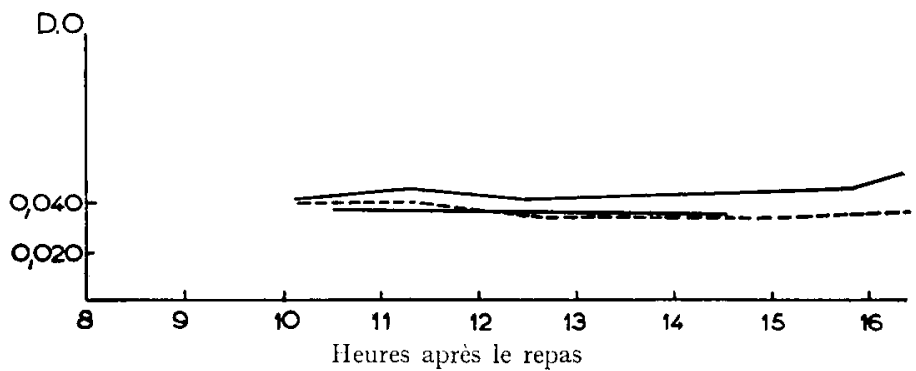

Fig. 7. - Évolution de la densité optique de différents sangs témoins, en fonction du temps écoulé depuis le repas. En ordonnées la densité optique par rapport au nitrite.

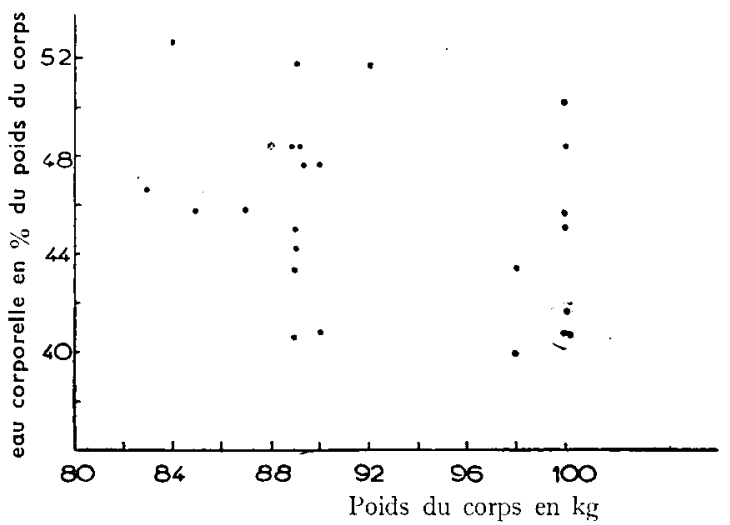

Fig. 8 